\title{
Improving the accuracy of AIRS by incorporating real world tournament selection in resource competition phase
}

\begin{abstract}
Artificial Immune Recognition System (AIRS) is an immune inspired classifier that competes with famous classifiers. One of the most important components of AIRS is resource competition. The goal of resource competition is the development of the fittest individuals. Resource competition phase removes weakest individuals and selects strongest (seemly good) individuals. This type of selection has high selective pressure with a loss of diversity. It may generate premature memory cells and decrease the accuracy of classifier. In this study, the Real World Tournament Selection (RWTS) method is incorporated in resource competition phase of AIRS to prevent this issue and experiments are conducted to evaluate the accuracy of new algorithm (RWTSAIRS). The combination of cross validation and t test is used as evaluation method. Algorithms tested on benchmark datasets of the UCI machine learning repository show that RWTSAIRS obtained higher accuracy than AIRS in all cases and that the difference between accuracies of two algorithms was significant in majority of cases.
\end{abstract}

Keyword: Artificial immune recognition system; AIRS; Resource competition phase; Real World Tournament Selection (RWTS); RWTSAIRS 